\title{
Kinetics of Serum Carnitine Fractions in Patients With Chronic Kidney Disease Not on Dialysis
}

\author{
JUNKO YANO, SAKUYA ITO, GOH KODAMA, YOSUKE NAKAYAMA, YUSUKE KAIDA, \\ YUNOSUKE YOKOTA, YUKIE KINOSHITA*, KYOKO TASHIRO* AND KEI FUKAMI \\ Division of Nephrology, Department of Medicine, \\ *Research Institute of Medical Mass Spectrometry, Kurume University School of Medicine, Kurume 830-0011, Japan
}

Received 29 July 2019, accepted 29 August 2019

J-STAGE advance publication 26 August 2020

Edited by TSUKASA IGAWA

\begin{abstract}
Summary: Background: Carnitine plays a pivotal role in energy synthesis through $\beta$-oxidation in mitochondria. Serum and tissue levels of free carnitine are significantly decreased in dialysis patients, whereas acylcarnitine levels are increased. However, the precise kinetics and fate of carnitine fractions in chronic kidney disease (CKD) patients who are not on dialysis have not been clarified. This study aims to determine the kinetics of serum carnitine fractions in patients who were not on dialysis.

Methods: Seventy-five CKD patients not on dialysis were recruited in this study. Serum and urinary carnitine fraction levels were measured to evaluate the kinetics and regulation of serum carnitine fractions. Carnitine fractions were measured by the enzymatic cycling method.

Results: Total and free serum carnitine levels did not change with progression of CKD, whereas acylcarnitine levels and the acyl/free carnitine ratio significantly increased. Serum acylcarnitine levels were inversely associated with estimated glomerular filtration rate $\left(r^{2}=0.239, p<0.001\right)$, but free carnitine levels were not. Serum free carnitine levels were positively associated with urinary free carnitine excretion $\left(\mathrm{r}^{2}=0.214, \mathrm{p}<0.001\right)$, but serum acylcarnitine levels were not. Multiple stepwise regression analysis revealed that urinary free carnitine excretion and blood urea nitrogen were independent determinants of serum free carnitine and acylcarnitine levels, respectively.

Conclusions: The present study demonstrated that serum acylcarnitine levels increased with renal dysfunction independent of urinary excretion levels. Serum free carnitine was not affected by renal function in CKD patients who were not on dialysis.
\end{abstract}

Key words free carnitine, acylcarnitine, CKD, renal excretion

\section{BACKGROUND}

Carnitine is a natural substance found in protein-rich foods. It is also synthesized in the liver, kidney, and several other tissues, and is excreted from the kidney if levels become excessive [1]. Carnitine forms free and high-energy ester bonds with short or long fatty acids, such as acylcarnitine. Free carnitine participates in fatty acid $\beta$-oxidation and energy production by transporting long-chain fatty acids from the cytoplasm to the mitochondria [2]. Further, free carnitine regulates the function of the mitochondrial respiratory chain and is capable of eliminating excess intracellular fatty acids in muscular and cardiac cells as acylcarnitine [2]. Therefore, acyl/free carnitine ratio in the serum or plasma is now widely used as a marker of

Corresponding Author: Kei Fukami, M.D., Ph.D., Division of Nephrology, Department of Medicine, Kurume University School of Medicine, 67 Asahimachi, Kurume, Fukuoka 830-0011, Japan. Tel: +81-942-31-7002, Fax: +81-942-31-7763, E-mail: fukami@med.kurume-u.ac.jp

Abbreviations: ANOVA, analysis of variance; BP, blood pressure; BUN, blood urea nitrogen; CKD, chronic kidney disease; eGFR, estimated glomerular filtration rate; HD, hemodialysis; HDL, high-density lipoprotein; LDL, low-density lipoprotein; NAFLD, non-alcoholic fatty liver disease; OCTN2, organic cation/carnitine transporter. 
tissue carnitine deficiency. Carnitine deficiency causes muscle weakness and cardiac hypertrophy in rodents and humans [3-5]. Since more than $60 \%$ of serum carnitine is eliminated from the blood by hemodialysis (HD) $[6,7]$, carnitine deficiency is often observed in patients with HD. It has been reported that HD-related loss of carnitine accelerates the progression of cardiovascular disease in uremic subjects with an increased acyl/free carnitine ratio [8].

Circulating carnitine is reported to be mainly distributed between two compartments, large and slow turnover (muscle), and small and rapid turnover (liver, kidney, and other tissues)[9]. In healthy subjects, carnitine is efficiently reabsorbed by the transporter organic cation/carnitine transporter (OCTN2) in proximal tubular cells located in the brush border of the proximal tubular cell and is tightly regulated. Since carnitine levels are reported to be maintained within the normal range in vegetarians, healthy subjects conserve carnitine through renal tubular reabsorption[9]. In kidney disorders such as Fanconi syndrome, severe carnitine deficiency is reported due to decreased reabsorption in the proximal tubular cells[10]. However, the concentration of carnitine levels in other chronic kidney disease (CKD) patients not on HD is controversial $[11,12]$. The main pathological renal involvement in patients with CKD is mitochondria-rich tubular cell dysfunction; therefore, we speculate that carnitine deficiency in subjects with CKD could induce tubular cell damage, leading to the progression of renal dysfunction. However, the precise fate and kinetics of circulating and urinary carnitine fraction have not yet been examined in CKD patients who are not on HD. In this study we measured the carnitine fraction levels in serum and urine and assessed the correlation with renal function and CKD staging.

\section{METHODS}

\section{Patients}

CKD patients who were not on HD were recruited in Kurume University Hospital (56.1 \pm 20.0 years old, 34 male and 41 female, diabetic nephropathy $(n=16)$, chronic glomerulonephritis $(\mathrm{n}=28)$, membranous glomerulonephritis $(\mathrm{n}=8)$, minimal-change nephrotic syndrome $(\mathrm{n}=10)$, lupus nephritis $(\mathrm{n}=3)$, hypertensive nephrosclerosis $(\mathrm{n}=2)$, polycystic kidney disease $(\mathrm{n}=1)$, interstitial nephritis $(\mathrm{n}=1)$, unknown etiology $(n=6))$. The diagnosis of CKD and its staging were determined in line with the National Kidney Foundation Kidney Disease Outcome Quality Initiative guidelines [13]. Informed consent was obtained from all patients, and the study protocol was approved by the institutional ethics committees of Kurume University School of Medicine (Approval Number; 12078). This work was conducted in line with the Declaration of Helsinki and was registered with the University Hospital Medical Information Network clinical trial database (UMIN000010953).

\section{Data Collection}

Medical history was obtained by questionnaire. Body mass index was calculated as an index of the presence or absence of obesity. Blood pressure (BP) was measured in an upright sitting position using a standard sphygmomanometer. Vigorous physical activity and smoking were avoided for at least $30 \mathrm{~min}$ utes before BP measurement.

Fasting blood was drawn from the antecubital vein to determine serum albumin, hemoglobin, blood urea nitrogen (BUN), creatinine, uric acid, and lipids (total cholesterol, high-density lipoprotein (HDL)-cholesterol, low-density lipoprotein (LDL)-cholesterol, and triglycerides). Spot urine was collected for the determination of proteinuria $(\mathrm{g} / \mathrm{gCr})$ and carnitine fractions. HDL-cholesterol and LDL-cholesterol were measured at a commercially available laboratory (Daiichi Pure Chemicals Co., Ltd, Tokyo, Japan). The other chemistries were measured at a commercially available laboratory (Wako Pure Chemical Industries, Ltd, Osaka, Japan). Serum and urinary levels of total and free carnitine, and acylcarnitine were determined using an enzymatic cycling method [14]. Urinary carnitine/creatinine ratio was used as an adjusted urinary carnitine fraction level.

Estimated glomerular filtration rate (eGFR) was calculated using the following formula; $\mathrm{eGFR}=0.741$ $\times 175 \times$ serum $\mathrm{Cr}^{-1.154} \times$ age $^{-0.203} \times(0.742$ if female $)$ $[15,16]$.

\section{Statistical Analysis}

Results are presented as mean \pm standard deviation. Univariate regression analysis was performed for determinants of serum and urinary free and acylcarnitine. Multiple stepwise regression analysis was performed to assess the independent determinants of serum level free and acylcarnitine. When we analyzed independent determinants of serum acylcarnitine, BUN was used as a marker of the renal function instead of serum creatinine and eGFR because BUN showed the strongest correlation with serum acylcarnitine. One-way analysis of variance (ANOVA) was used to compare the urinary and serum carnitine levels among CKD stages. If the data met the assumption of 
homogeneity of variances, Tukey's honestly significant difference post hoc test was performed. Statistical significance was defined as $p<0.05$. All statistical analyzes were performed with SPSS version 20 software.

\section{RESULTS}

\section{Demographic Data}

Demographic data of the patients are presented in Table 1. Average serum levels of total, free, and acylcarnitine were $57.8 \pm 17.4,45.0 \pm 13.5$, and $12.8 \pm$ $7.8 \mu \mathrm{mol} / \mathrm{L}$, respectively. Acyl/free carnitine ratio was $0.30 \pm 0.19$.

Correlation between serum carnitine fraction levels and renal function

Serum acylcarnitine levels and acyl/free carnitine ratio were inversely correlated with eGFR $\left(r^{2}=\right.$ $0.239, \mathrm{p}<0.001, \mathrm{r}^{2}=0.290, \mathrm{p}<0.001$, respectively), whereas total and free carnitine levels were not (Fig. 1A-D). CKD stage did not affect serum total and free carnitine levels (Fig. 2A and B). On the other hand, serum acylcarnitine levels and acyl/free carnitine ratio were significantly increased in patients with CKD stage G5 compared with those in CKD stages G1-3 (Fig. 2C and D).

\section{Correlation between urinary carnitine fraction levels and renal function}

Next we evaluated the relationship between urinary carnitine fraction levels and renal function in these patients. As shown in Fig. 3, urinary free carnitine and acylcarnitine levels were not associated with renal function (Fig. 3A and B). Further, there was no difference in either urinary carnitine fraction according to the CKD stages (Fig. 3C and D).

\section{Independent determinants of serum free carnitine and acylcarnitine}

Next we evaluated the independent determinants of serum free carnitine and acylcarnitine in these patients. Urinary free carnitine levels were positively associated with serum free carnitine $\left(\mathrm{r}^{2}=0.214, \mathrm{p}<\right.$ 0.001 ) (Fig. 4A), and were an independent determinant of serum free carnitine (Table 2). In contrast, urinary acylcarnitine levels were not associated with serum acylcarnitine levels. Several variables such as sex, hemoglobin, eGFR, total cholesterol, and HDLcholesterol were all inversely associated with serum acylcarnitine values, whereas BUN, serum creatinine,
TABLE 1.

Clinical characteristics of the patients

\begin{tabular}{ll}
\hline Variables & Mean $\pm \mathrm{SD}$ \\
\hline No. of patients & 75 \\
Age (years old) & $56.1 \pm 20.0$ \\
Sex (No.) (male/female) & $34 / 41$ \\
Body weight $(\mathrm{kg})$ & $59.6 \pm 13.6$ \\
Body mass index $\left(\mathrm{kg} / \mathrm{m}^{2}\right)$ & $23.3 \pm 4.3$ \\
Mean blood pressure $(\mathrm{mmHg})$ & $97.2 \pm 12.1$ \\
Hemoglobin $(\mathrm{g} / \mathrm{dl})$ & $11.1 \pm 2.6$ \\
Serum albumin $(\mathrm{g} / \mathrm{dl})$ & $2.90 \pm 0.96$ \\
Blood urea nitrogen $(\mathrm{mg} / \mathrm{dl})$ & $43.0 \pm 29.7$ \\
Serum creatinine $(\mathrm{mg} / \mathrm{dl})$ & $3.45 \pm 3.03$ \\
eGFR $\left(\mathrm{ml} / \mathrm{min} / 1.73 \mathrm{~m}{ }^{2}\right)$ & $38.1 \pm 37.7$
\end{tabular}

No. of the patients (according to eGFR)

$\begin{array}{ll}\geqq 90 & 9 \\ 60-<90 & 12 \\ 30-<60 & 9 \\ 15-<30 & 14 \\ <15 & 31\end{array}$

UP/UCrea $(\mathrm{g} / \mathrm{gCr})$

$5.48 \pm 5.21$

Uric acid (mg/dl)

$7.25 \pm 2.29$

Total cholesterol (mg/dl)

$224 \pm 99$

LDL-cholesterol (mg/dl)

$139 \pm 82$

HDL-cholesterol (mg/dl)

$52 \pm 24$

Triglycerides a $(\mathrm{mg} / \mathrm{dl})$

164 (56-694)

Total carnitine $(\mu \mathrm{mol} / \mathrm{l})$

$57.8 \pm 17.4$

Free carnitine $(\mu \mathrm{mol} / \mathrm{l})$

$45.0 \pm 13.5$

Acylcarnitine $(\mu \mathrm{mol} / \mathrm{l})$

$12.8 \pm 7.8$

Acyl/Free carinitine ratio

$0.30 \pm 0.19$

UFreeC/UCrea $\quad(\mathrm{mmol} / \mathrm{molCr})$

$16.7 \pm 27.2$

UAcylC/UCrea $\quad(\mathrm{mmol} / \mathrm{molCr})$

$12.2 \pm 8.2$

Diabetes (No.) $(-/+)$

Values are shown as mean \pm SD or range. $\mathrm{SD}=$ standard deviation; No.=number; eGFR=estimated glomerular filtration rate; UP/UCrea=urinary protein/urinary creatinine ratio; LDL=low-density lipoprotein; HDL=high-density lipoprotein; UFreeC/UCrea=urinary free carnitine/urinary creatinine ratio; UAcylC/Ucrea=urinary acylcarnitine/

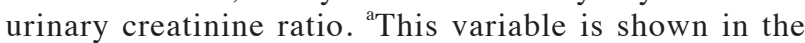
original scale after using ln-transformed values. 
and uric acid had a positive association. BUN was the sole independent determinant of serum acylcarnitine in these patients $(\mathrm{p}<0.001)$ (Fig. 4B) (Table 3$)$.

\section{DISCUSSION}

The salient findings of the present study are as follows. First, serum total and free carnitine levels did not change with progression of CKD, whereas acylcarnitine and acyl/free carnitine ratio were significantly increased in patients with CKD stage G5. Second, urinary free carnitine values are an independent correlate of serum free carnitine in CKD patients. Third, renal dysfunction evaluated by BUN was an independent determinant of serum acylcarnitine; however, urinary acylcarnitine levels were not correlated with serum acylcarnitine in these patients. These observations suggest that serum free carnitine might be strictly regulated by urinary free carnitine excretion, but serum acylcarnitine might increase in uremic conditions independent of renal excretion.

It has been reported that serum carnitine levels increase significantly in patients with renal failure. Chen et al. showed that serum carnitine levels were higher in patients with kidney disease compared with healthy subjects [17]. Further, they reported that patients with oliguria showed a large increase in serum carnitine, and concluded that the clinical significance of increased carnitine in renal insufficiency might be analogous to that of BUN, serum creatinine, or uric acid [17]. However, they did not measure free carnitine and acylcarnitine separately and did not report the correlation between serum and urinary carnitine fractions. In this study, acylcarnitine levels, but not free carnitine levels, were positively correlated with renal function, and independently associated with BUN levels, thus, the main increased carnitine fraction in renal insufficiency might be acylcarnitine. Further, serum free carnitine values were positively associated with urinary free carnitine levels, in that urinary free carnitine was a sole independent determinant of serum free carnitine, suggesting that serum free carnitine levels might tightly be regulated by the OCTN2 carnitine transporter in proximal tubular cells, thereby maintaining circulating free carnitine levels even in CKD patients who are not on HD.

Acylcarnitine levels have been reported to be higher in patients with renal insufficiency due to its decreased excretion in urine. In our study, serum acylcarnitine values also increased according to renal dysfunction; however, serum and urinary acylcarnitine levels were not correlated. Therefore, serum acylcarnitine levels increase with renal insufficiency independent of renal dysfunction. From these findings, we
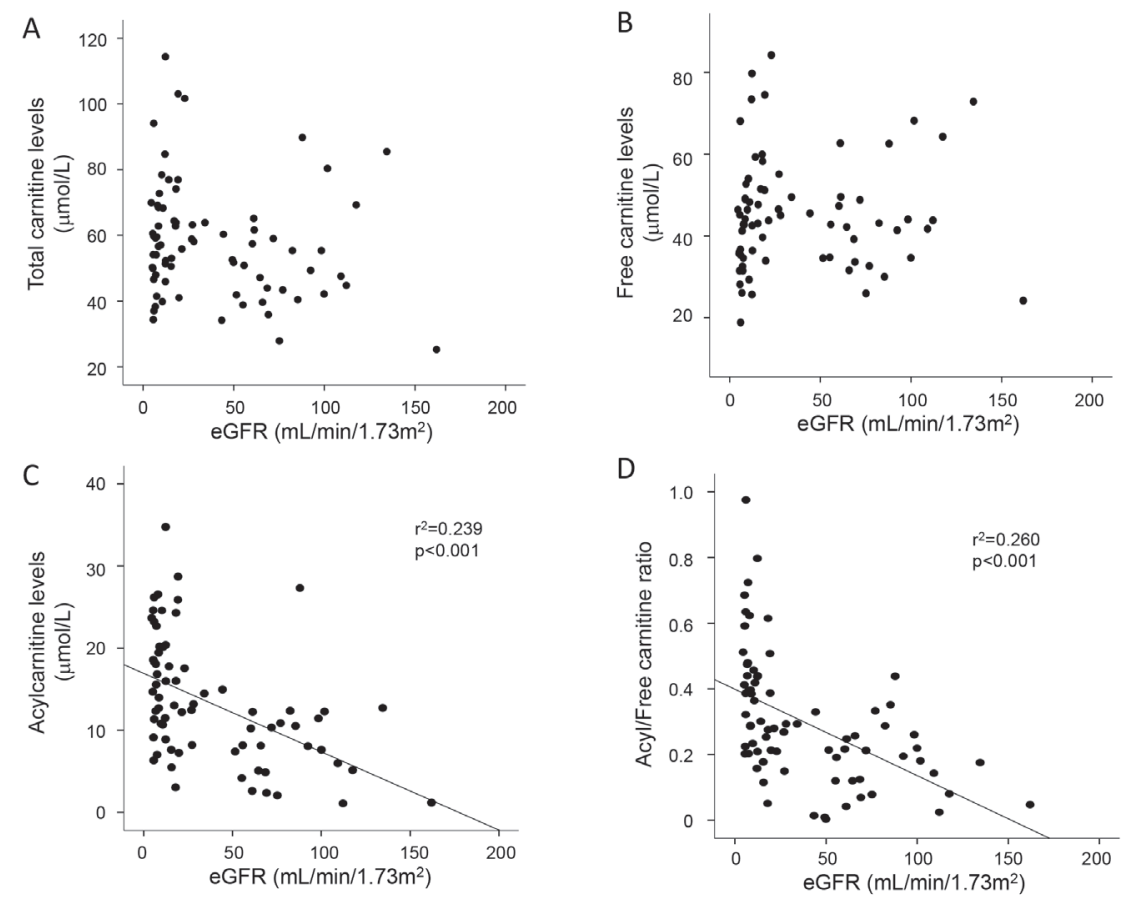

Fig. 1. Linear regression line of serum total carnitine (A), free carnitine (B), acylcarnitine (C), acyl/free carnitine ratio (D) and eGFR in patients with CKD who were not on dialysis. eGFR: estimated glomerular filtration ratio, CKD: chronic kidney disease. 
A

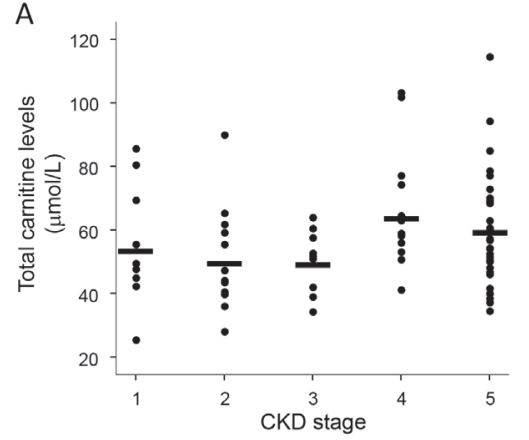

C

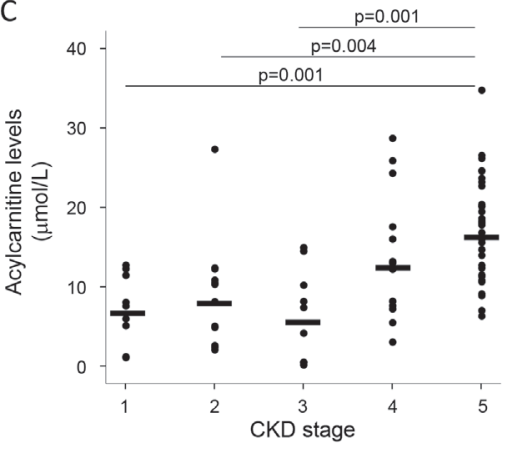

B
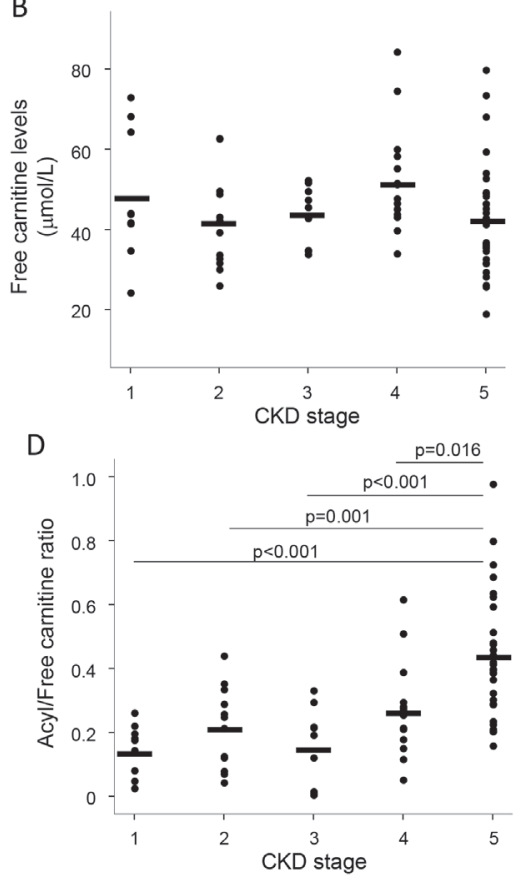

Fig. 2. Serum total carnitine (A), free carnitine (B), acylcarnitine (C), and acyl/ free carnitine ratio (D) levels according to CKD stage in patients with CKD who were not on dialysis. eGFR: estimated glomerular filtration ratio, CKD: chronic kidney disease.

A

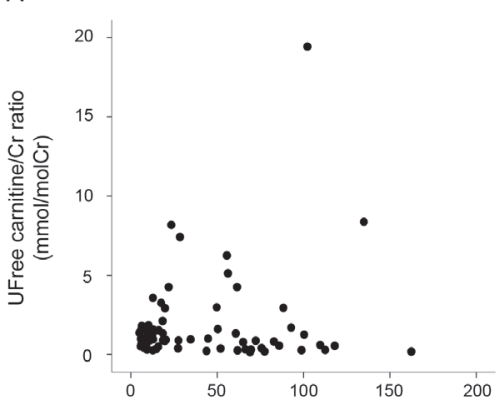

C

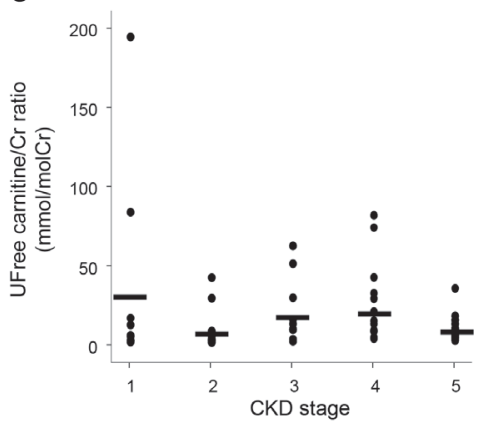

B

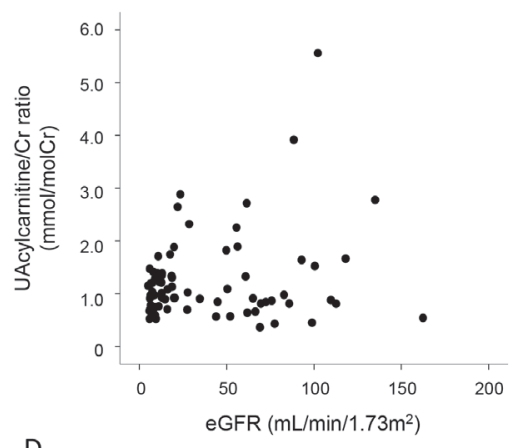

D

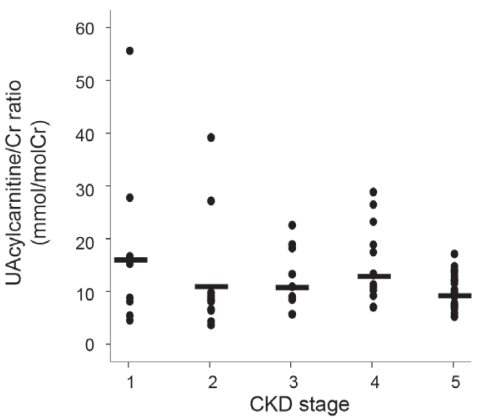

Fig. 3. Association between urinary carnitine fractions and renal function.

Relationship between eGFR and UFree carnitine/Cr ratio (A) and UAcylcarnitine/Cr ratio (B). UFree carnitine/Cr ratio (C) and UAcylcarnitine/Cr ratio (D) according to CKD stage in patients with $\mathrm{CKD}$ who were not on dialysis. UFree carnitine/Cr ratio: urinary free carnitine/urinary creatinine ratio, UAcylcarnitine/Cr ratio: urinary acylcarnitine/urinary creatinine ratio, eGFR: estimated glomerular filtration ratio, CKD: chronic kidney disease. 
A

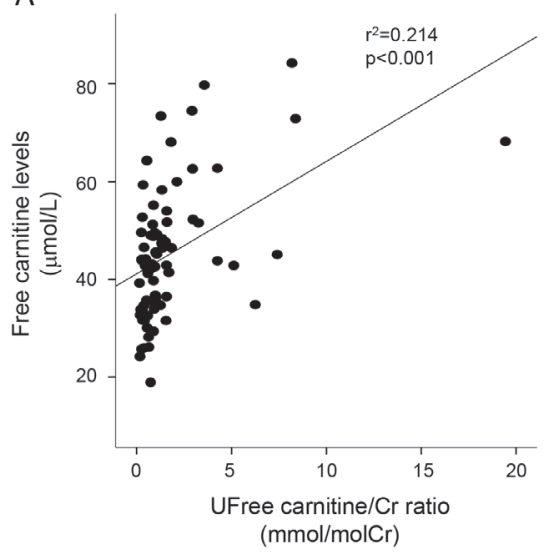

B

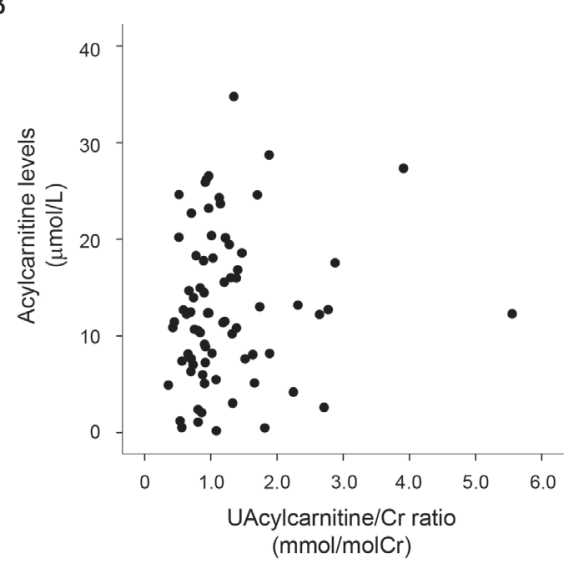

Fig. 4. Association between urinary and serum carnitine fractions.

Relationship between serum free carnitine levels and UFree carnitine/Cr ratio (A) and serum acylcarnitine levels and UAcylcarnitine/Cr ratio (B) in patients with $\mathrm{CKD}$ who are not on dialysis. UFree carnitine/Cr ratio: urinary free carnitine/urinary creatinine ratio, UAcylcarnitine/Cr ratio: urinary acylcarnitine/urinary creatinine ratio, CKD: chronic kidney disease.

TABLE 2

Univariate and multiple stepwise regression analysis for the determinants of serum free carnitine levels

\begin{tabular}{|c|c|c|c|c|c|c|}
\hline \multirow[b]{2}{*}{ Variables } & \multicolumn{3}{|c|}{ Univariate regression } & \multicolumn{3}{|c|}{ Multiple regression } \\
\hline & SE & $\beta$ & $\mathrm{p}$ & SE & $\beta$ & $\mathrm{p}$ \\
\hline Age & .080 & -.158 & 0.177 & & & \\
\hline Sex & 3.152 & -.081 & 0.489 & & & \\
\hline Body weight & .116 & .065 & 0.578 & & & \\
\hline Body mass index & .371 & .059 & 0.616 & & & \\
\hline Mean blood pressure & .131 & -.074 & 0.528 & & & \\
\hline Hemoglobin & .599 & .182 & 0.118 & & & \\
\hline Serum albumin & 1.647 & -.060 & 0.611 & & & \\
\hline Blood urea nitrogen & .053 & .010 & 0.934 & & & \\
\hline Serum creatinine & .515 & -.171 & 0.143 & & & \\
\hline eGFR & .042 & .028 & 0.812 & & & \\
\hline UP/UCrea & .304 & -.057 & 0.629 & & & \\
\hline Uric acid & .690 & .024 & 0.837 & & & \\
\hline Total cholesterol & .016 & .084 & 0.473 & & & \\
\hline LDL_cholesterol & .019 & .088 & 0.453 & & & \\
\hline HDL-cholesterol & .065 & -.078 & 0.505 & & & \\
\hline Triglycerides & 3.124 & .157 & 0.178 & & & \\
\hline UFreeC/UCrea & .052 & 0.463 & $<0.001$ & .052 & 0.463 & $<0.001$ \\
\hline Diabetes & 3.455 & .034 & 0.772 & & & \\
\hline
\end{tabular}

$\mathrm{R}^{2}=0.149$. eGFR=estimated glomerular filtration rate; $\mathrm{UP} / \mathrm{UCrea}=$ urinary protein/urinary creatinine ratio; LDL=low-density lipoprotein; HDL=high-density lipoprotein; UFreeC/UCrea=urinary free carnitine/urinary creatinine ratio. 
TABLE 3.

Univariate and multiple stepwise regression analysis for the determinants of serum acylcarnitine levels

\begin{tabular}{|c|c|c|c|c|c|c|}
\hline \multirow[b]{2}{*}{ Variables } & \multicolumn{3}{|c|}{ Univariate regression } & \multicolumn{3}{|c|}{ Stepwise regression } \\
\hline & $\mathrm{SE}$ & $\beta$ & $\mathrm{p}$ & SE & $\beta$ & $\mathrm{p}$ \\
\hline Age & .046 & -.166 & 0.154 & & & \\
\hline Sex & 1.730 & -.308 & 0.007 & & & \\
\hline Body weight & .066 & .162 & 0.164 & & & \\
\hline Body mass index & .213 & .086 & 0.463 & & & \\
\hline Mean blood pressure & .075 & .052 & 0.659 & & & \\
\hline Hemoglobin & .340 & -.238 & 0.040 & & & \\
\hline Serum albumin & .095 & .094 & 0.421 & & & \\
\hline Blood urea nitrogen & .026 & .530 & $<0.001$ & .026 & .530 & $<0.001$ \\
\hline Serum creatinine & .261 & .496 & $<0.001$ & & & \\
\hline eGFR & .021 & -.477 & $<0.001$ & & & \\
\hline UP/UCrea & .173 & -.147 & 0.210 & & & \\
\hline Uric acid & .356 & .442 & $<0.001$ & & & \\
\hline Total cholesterol & .009 & -.233 & 0.044 & & & \\
\hline LDL-cholesterol & .011 & -.158 & 0.175 & & & \\
\hline HDL-cholesterol & .036 & -.321 & 0.005 & & & \\
\hline Triglycerides & 1.811 & -.094 & 0.424 & & & \\
\hline UAcylC/UCrea & .110 & .104 & 0.375 & & & \\
\hline Diabetes & 1.960 & .165 & 0.157 & & & \\
\hline
\end{tabular}

$\mathrm{R}^{2}=0.281$. eGFR=estimated glomerular filtration rate; UP/UCrea=urinary protein/urinary creatinine ratio; LDL=low-density lipoprotein; HDL=high-density lipoprotein; UAcylC/UCrea=urinary acylcarnitine/urinary creatinine ratio.

speculate that some uremic toxins or mitochondrial dysfunction that results from renal dysfunction might cause the accumulation of acylcarnitine in serum in CKD patients. Since long-chain acylcarnitine in the serum has been reported, from metabolome analysis, to be a marker of cardiovascular death in HD patients [18], evaluating serum acylcarnitine levels might be valuable in maintaining the patient's quality of life.

In this study, the acyl/free carnitine ratio also increased in association with the progression of renal dysfunction. A higher acyl/free carnitine ratio reflects tissue carnitine deficiency and decreased energy synthesis in mitochondria. In addition, it is associated with increased cardiac event rates in patients with heart failure [19]. Therefore, a higher acyl/free carnitine ratio might reflect carnitine deficiency in tissue and, consequently, may be involved in the development of cardiovascular disease in patients with CKD.

A recent study reported a close association be- tween the serum acylcarnitine profile and non-alcoholic fatty liver disease (NAFLD) and NAFLD-related hepatocellular carcinoma [20]. Further, serum C16-acylcarnitine levels were affected by lung cancer [21]. Although we did not measure the serum acylcarnitine profile in this study, an examination of longchain acylcarnitine might be valuable for evaluating the prognosis of patients with CKD.

Serum free carnitine levels less than $20 \mu \mathrm{mol} / \mathrm{L}$ are a criterion for carnitine deficiency. However, in our study, serum free carnitine levels in almost all patients were over $20 \mu \mathrm{mol} / \mathrm{L}$, which differed from patients who underwent HD. Therefore, the evaluation of tissue carnitine levels, such as in red blood cells, might be useful to understand intracellular carnitine metabolism.

In conclusion, the present study demonstrates that serum levels of acylcarnitine increased according to the progression of CKD independent of urinary acyl- 
carnitine levels. These findings suggest that serum acylcarnitine may be regulated by a renal dysfunction-associated uremic condition in CKD patients who are not on dialysis.

ACKNOWLEDGMENT: This work was supported in part by a Grant-in-Aid for Welfare, and Scientific Research (C) (No. 19K08693) from the Ministry of Education, Culture, Sports, Science and Technology of Japan (KF).

\section{DECLARATIONS}

\section{ETHICS APPROVAL AND CONSENT TO}

PARTICIPATE: Informed consent was obtained from all patients, and the study protocol was approved by the Institutional Ethics Committee of Kurume University School of Medicine (Approval Number; 12078).

\section{CONSENT FOR PUBLICATION: Not applicable.}

AVAILABILITY OF DATA AND MATERIAL: The datasets used and analyzed during the current study are available from the corresponding author on reasonable request.

COMPETING INTERESTS: KF has received honoraria such as lecture fees from Otsuka Pharmaceutical Co., Ltd.

FUNDING: Not applicable.

\section{REFERENCES}

1. Wanner $\mathrm{C}$ and Hörl WH. Carnitine abnormalities in patients with renal insufficiency. Pathophysiological and therapeutical aspects. Nephron 1988; 50(2):89-102.

2. Evans AM and Fornasini G. Pharmacokinetics of L-carnitine. Clin Pharmacokinet 2003; 42(11):941-967.

3. Knapp AC, Todesco L, Torok M, Beier K, and Krähenbühl S. Effect of carnitine deprivation on carnitine homeostasis and energy metabolism in mice with systemic carnitine deficiency. Ann Nutr Metab 2008; 52(2):136-144.

4. Carvajal K and Moreno-Sánchez R. Heart metabolic disturbances in cardiovascular diseases. Arch Med Res 2003; 34(2):89-99.

5. Ascunce RR, Nayar AC, Phoon CK, and Srichai MB. Cardiac magnetic resonance findings in a case of carnitine deficiency. Tex Heart Inst J 2013; 40(1):104-105.

6. Evans A. Dialysis-related carnitine disorder and levocarnitine pharmacology. Am J Kidney Dis 2003; 41(4 Suppl 4):S13-S26.

7. Adachi T, Fukami K, Yamagishi S, Kaida Y, Ando R et al. Decreased serum carnitine is independently correlated with increased tissue accumulation levels of advanced glycation end products in haemodialysis patients. Nephrology (Carlton) 2012; 17(8):689-694 .
8. Pauly DF and Pepine CJ. The role of carnitine in myocardial dysfunction. Am J Kidney Dis 2003; 41(4 Suppl 4):S35S43.

9. Rebouche CJ. Kinetics, pharmacokinetics, and regulation of L-carnitine and acetyl-L-carnitine metabolism. Ann N Y Acad Sci 2004; 1033:30-41.

10. Bernardini I, Rizzo WB, Dalakas M, Bernar J, and Gahl WA. Plasma and muscle free carnitine deficiency due to renal Fanconi syndrome. J Clin Invest 1985; 75(4):11241130.

11. Wanner C, Förstner-Wanner S, Rössle C, Fürst $P$, Schollmeyer $\mathrm{P}$ et al. Carnitine metabolism in patients with chronic renal failure: effect of L-carnitine supplementation. Kidney Int Suppl 1987; 22:S132-S135.

12. Fouque D, Holt S, Guebre-Egziabher F, Nakamura K, Vianey-Saban $\mathrm{C}$ et al. Relationship between serum carnitine, acylcarnitines, and renal function in patients with chronic renal disease. J Ren Nutr 2006; 16(2):125-131.

13. Levey AS, Eckardt KU, Tsukamoto Y, Levin A, Coresh J et al. Definition and classification of chronic kidney disease: a position statement from Kidney Disease: Improving Global Outcomes (KDIGO). Kidney Int 2005; 67(6):20892100.

14. Fukami K, Yamagishi S, Sakai K, Kaida Y, Minami A et al. Carnitine deficiency is associated with late-onset hypogonadism and depression in uremic men with hemodialysis. Aging Male 2014; 17(4):238-242.

15. Imai $E$, Horio $M$, Nitta $K$, Yamagata $K$, Iseki $K$ et al. Modification of the Modification of Diet in Renal Disease (MDRD) Study equation for Japan. Am J Kidney Dis 2007; 50(6):927-937.

16. Levey AS, Coresh J, Greene T, Stevens LA, Zhang YL et al. Using standardized serum creatinine values in the modification of diet in renal disease study equation for estimating glomerular filtration rate. Ann Intern Med 2006; 145(4):247-254.

17. Chen SH and Lincoln SD. Increased serum carnitine concentration in renal insufficiency. Clin Chem 1977; 23(2 PT. 1):278-280.

18. Kalim S, Clish CB, Wenger J, Elmariah S, Yeh RW et al. A plasma long-chain acylcarnitine predicts cardiovascular mortality in incident dialysis patients. J Am Heart Assoc 2013; 2(6):e000542.

19. Yoshihisa A, Watanabe S, Yokokawa T, Misaka T, Sato T et al. Associations between acylcarnitine to free carnitine ratio and adverse prognosis in heart failure patients with reduced or preserved ejection fraction. ESC Heart Fail 2017; 4(3):360-364.

20. Enooku K, Nakagawa H, Fujiwara N, Kondo M, Minami T et al. Altered serum acylcarnitine profile is associated with the status of nonalcoholic fatty liver disease (NAFLD) and NAFLD-related hepatocellular carcinoma. Sci Rep 2019; 9(1): 10663.

21. Ni J, Xu L, Li W, Zheng C, and Wu L. Targeted metabolomics for serum amino acids and acylcarnitines in patients with lung cancer. Exp Ther Med 2019; 18(1):188-198. 